\title{
Corrigendum
}

\section{Increased consumption of calcium from fat-free milk, energy-restricted diet and educational activities improves metabolic control in overweight type 2 diabetic patients - CORRIGENDUM}

Jorge de Assis Costa, Júnia Maria Geraldo Gomes, Priscila Vaz de Melo Ribeiro and Rita de Cássia Gonçalves Alfenas

(First published online $9^{\text {th }}$ December 2019)

doi: $10.1017 /$ S0007114519003192

\section{Original text}

Jorge de Assis Costa ${ }^{1}$, Júnia Maria Geraldo Gomes ${ }^{1}$, Priscila Vaz de Melo Ribeiro ${ }^{2 *}$ and Rita de Cássia Gonçalves Alfenas ${ }^{2}$ ${ }^{1}$ Instituto Federal do Sudeste de Minas Gerais (UEMG) - Campus Barbacena, Barbacena, Minas Gerais CEP 36205-018, Brazil ${ }^{2}$ Departamento de Nutrição e Saúde, Universidade Federal de Viçosa, Viçosa, Minas Gerais CEP 36570-000, Brazil

\section{Should be, corrected}

Jorge de Assis Costa ${ }^{1}$, Júnia Maria Geraldo Gomes ${ }^{2}$, Priscila Vaz de Melo Ribeiro ${ }^{3 *}$ and Rita de Cássia Gonçalves Alfenas ${ }^{3}$

${ }^{1}$ Universidade do Estado de Minas Gerais (UEMG) - Campus Barbacena, Minas Gerais CEP 36202-284, Brazil ${ }^{2}$ Instituto Federal do Sudeste de Minas Gerais - Campus Barbacena, Barbacena, Minas Gerais CEP 36205-018, Brazil

${ }^{3}$ Departamento de Nutrição e Saúde, Universidade Federal de Viçosa, Viçosa, Minas Gerais CEP 36570-000, Brazil 